\title{
Alcune formole relative alle linee tracciate sopra una superficie e loro applicazioni.
}

\author{
(Di Geminuno Pirondin, a Parma.)
}

$\S 1$.

Siano $u=$ cost. $^{\mathrm{e}}, v=$ cost. $^{\mathrm{e}}$ le equazioni delle linee di curvatura di una superficie $S$ non sviluppabile, ed $L$ una linea qualunque tracciata sopra $S$, segante le linee $v=$ cost." sotto l'angolo $i$. Chiamando $\rho_{n}$ il raggio della sezione normale tangente ad $L, e r_{u}, r_{v}$ i raggi di curvatura principali della $S$ relativi alle linee $u=$ cost. $^{e}, v=$ cost. $^{e}$, si ha

$$
\frac{1}{\rho_{n}}=\frac{\operatorname{sen}^{2} i}{r_{i}}+\frac{\cos ^{2} i}{r_{v}} \text {. }
$$

Se poi $\theta$ è l'angolo sotto il quale il piano osculatore di $L$ sega il piano tangente e $p$ il raggio di curvatura di $L$, si ha dal teorema di Meunier

$$
\frac{1}{p_{n}}=\frac{\operatorname{sen} 0}{p} \text {. }
$$

Osservando quindi che $\frac{\rho}{\cos \theta}$ rappresenta il raggio di curvatura geodetica di $L$ sopra la superficie $S$, si ricava dalle due relazioni precedenti

$$
\frac{1}{p^{2}}=\frac{1}{p^{2} y}+\left(\frac{\operatorname{sen}^{2} i}{r_{u}}+\frac{\cos ^{2} i}{r_{v}}\right)^{2}
$$

cioi, indicando con $H$ il raggio di curvatura della geodetica tangente ad $L$,

$$
\frac{1}{\rho^{2}}=\frac{1}{p^{2} g}+\frac{1}{H^{2}} \text {. }
$$

La quantità $\frac{1}{\rho^{2}}-\frac{1}{H^{2}}$ ¿̀ dunque un invariante di flessione della superficie. 
Chiamando $L_{1}$ una linea tangente ad $L$ in un punto e tracciata sulla stessa superficie $S$, si ha

$$
\frac{1}{\hat{p}^{2}}=\frac{1}{\hat{p}^{2}+g}+\frac{1}{H^{2}} \text {. }
$$

$\mathrm{E}$ se fra questa equazione e la precedente eliminiamo $H$, si ricava:

$$
\frac{1}{p^{2}}-\frac{1}{p^{2}}=\frac{1}{p^{2} g}-\frac{1}{p^{2} s g} \text {. }
$$

La quantità $\frac{1}{p^{2}}-\frac{1}{p^{2}}$, relativa al punto di contatto delle due linee tangenti, è dunque un invariante di flessione della superficie data.

Qualora poi la superficie sia sviluppabile, le equazioni (1), (2) divengono più semplici; supposto che le linee $v=$ cost. $^{e}$ siano le generatrici rettilinee, si ha $\frac{1}{r_{v}}=0$ e le equazioni (1), (2) forniscono la relazione

$$
\frac{1}{f^{2}}=\frac{1}{\rho^{2} g}+\frac{\operatorname{sen}^{4} i}{r^{2} t} \text {. }
$$

$\S 2$.

Se la sviluppabile è cilindrica, $r_{u}$ è eguale al raggio di curvatura $\rho_{0}$ della sezione retta $L_{0}$. Osservando poi che $\rho_{g}$ viene ad essere il raggio di curvatura della linea piana a cui si riduce $L$ collo sviluppo del cilindro, si ha

essendo $s_{0}$ l'arco di $L_{0}$.

$$
\frac{1}{p g}=\frac{d i}{d s}=\operatorname{sen} i \cdot \frac{d i}{d s o}
$$

Avremo dunque dalla (3)

$$
\frac{1}{p}=\operatorname{sen}^{2} i \sqrt{\frac{1}{p^{2}+}+\left(\frac{1}{\operatorname{sen} i} \frac{d i}{d s}\right)^{2}}
$$

e se rammentiamo che per la torsione $\frac{1}{r}$ di una linea tracciata sopra una sviluppabile si ha l'espressione

dove

$$
\frac{1}{r}=\frac{d \theta}{d s}-\frac{\operatorname{sen} \theta}{i} \cot i,
$$

$$
\cos \theta=\frac{\hat{i}}{p_{g}},
$$


avremo

$$
\frac{1}{\gamma}=\frac{\frac{1}{\operatorname{sen} i}\left(\frac{d i}{d s_{0}}\right)^{2}}{\frac{1}{\rho^{2} 0}+\left(\frac{1}{\operatorname{sen} i} \frac{d i}{d s_{0}}\right)^{2}} \cdot \frac{d}{d s_{0}}\left(\frac{\operatorname{sen} i}{\rho_{0} \frac{d i}{d s_{0}}}\right)-\frac{1}{\rho_{0}} \operatorname{sen} i \cdot \cos i .
$$

Consideriamo ad es. le curve cilindriche nelle quali

$$
\frac{i g}{i_{0}} \operatorname{sen}^{2} i=\frac{1}{k}
$$

con $l$ costante. Siccome da questa relazione si ricava

$$
\frac{\operatorname{sen} i}{d i}=\frac{1}{d s_{0}}
$$

le equazioni (4), (5) ci dànno

$$
\rho=\frac{1}{\sqrt{1+k^{2}}} \cdot \frac{\rho_{0}}{\operatorname{sen}^{2} i}, \quad r=-\frac{\rho_{0}}{\operatorname{sen} i \cos i} .
$$

Perciò: Se in ogni punto della linea cilindrica $L$ per la quale è soddisfatta la condizione (6), si conduce l'elica tangente l, iraggi di curvatura e quelli $d i$ torsione di tutte queste eliche $l$ nei punti di contatto, sono rispettivamente proporzionali ed eguali ai raggi di curvatura e di torsione di L nei medesimi punti.

La costruzione della linea. $L$ avente questa proprietà può effettuarsi col mezzo dell' equazione

$$
\cot i=\frac{1}{2 a}\left(a^{2} e^{k \int \frac{d s_{n}}{p_{n}}}-e^{-k \int \frac{d s_{n}}{p_{0}}}\right)
$$

che si deduce integrando la (7).

Se consideriamo in secondo luogo la curva $L$ che sul cilindro è una didonia, si ha

$$
\frac{1}{p_{y}}=\operatorname{sen} i \frac{d i}{d s_{0}}=\frac{1}{b}
$$

con $l i$ costante. Perciò per tale linea le equazioni (4), (5) divengono $\frac{1}{\rho}=\frac{\sqrt{k^{2} \rho_{0}+\left(k^{2}-s^{2}\right)^{2}}}{k_{k^{2} \rho_{0}}}, \frac{1}{r}=\frac{k \rho_{0}^{2} \sqrt{k^{2}-s^{2}}}{k^{2} \rho^{2} 0+\left(k^{2}-s^{2}\right)^{2}} \cdot \frac{d}{d s_{0}}\left(\frac{k^{2}-s^{2} 0}{k \rho_{0}}\right)+\frac{s_{0} \sqrt{k_{0}^{2}-s_{0}^{2}}}{k^{2} \rho_{0}}$.

Si vede quindi ad es. che la didonia cilindrica avrì curvatura assoluta co- 
stante $=\frac{1}{h}$ sempre e soltanto quando la sezione retta del cilindro sia la curva rappresentata dall' equazione

$$
\rho_{0}=\frac{h}{k \sqrt{k^{2}-h^{2}}} \cdot\left(k^{2}-s^{2}{ }_{0}\right)
$$

\section{$\S 3$.}

La $S$ sia una superficie conica e chiamiamo $R$ le porzioni delle generatrici rettilinee comprese fra il vertice e la curva $L$.

Osservando allora che $\rho_{g}$ rappresenta il raggio di curvatura assoluta della trasformata di $L$ quando il cono si sviluppa in un piano, si avrà $\left(^{*}\right)$

$$
P_{g}==\frac{R \sqrt{1-R^{\prime 2}}}{1-R_{i}^{\prime 2}-R R^{\prime \prime}},
$$

essendo le derivate di $R$ prese rapporto all'arco. Si osservi di più che $r_{\imath \iota}$ è eguale al raggio di curvatura geodetica, sulla sfera che la contiene, della linea di curvatura sferica del cono, passante pel punto di $L$ che si considera; e perciò

$$
r_{n}=R_{\tau},
$$

escendo : il raggio di curvatura geodetica della curva $\Lambda$ che si ottiene scgando il cono colla sfera di raggio 1 che ha il centro nel vertice.

Avremo perciò

$$
\frac{1}{\rho^{2}}=\frac{1}{l^{2}}\left\{\frac{1-R^{\prime 2}-R R^{\prime \prime}}{1-R^{\prime 2}}+\frac{\left(1-R^{\prime 2}\right)^{2}}{\tau^{2}}\right\}
$$

Se ad es. si considera una geodetica del cono, si ha

e quindi

$$
1-R^{\prime 2}-R R^{\prime \prime}=0
$$

$$
P=\frac{R \tau}{1-R_{i}^{\prime 2}}
$$

Percio: Il raggio di curvatura in un punto qualunque di una geodetica d'un cono è eguale al raggio di curvatura geodetica, sulla sfera che la contiene,

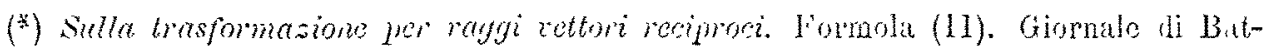
taglini, vol. AXVII. 
della linea di curvatura sferica del cono in quel punto, diviso per il quadrato del seno dell' inclinazione della geodetica sulla generatrice rettilinea del cono.

$\mathrm{Nel}$ caso-limite in cui il cono divenga un cilindro, si ha un teorema noto relativo alle eliche.

Se insieme all' equazione (9) si considera l'altra

$$
\frac{d s}{d s}=\frac{\sqrt{1-R^{2}}}{l}
$$

che lega l'arco elementare $d s$ di $L$ all'arco elementare $d \sigma$ della linea sferica $\Lambda$, si giunge all'importante conclusione che è costruibile in un sol modo la curva dello spazio di cui sono dati in funzione dell'areo il raggio di curvatura e il raggio vettore relativo a un punto fisso qualunque.

Esempi. 1.') Si voglia la carva determinata dalle equazioni

$$
R=s \cdot \cos i, \quad p=a s,
$$

con $i, a$ costanti. Dall' equazione (9) si ricava

$$
\frac{1}{\tau}=\frac{1}{1-R^{\prime 2}} \sqrt{\frac{R^{2}}{\rho^{2}}-\frac{\left(1-R^{2}-R R^{\prime \prime}\right)^{2}}{1-R^{\prime 2}}}
$$

e questa, nel caso nostro, ci dà

$$
\frac{1}{\tau}=\frac{\sqrt{\cos ^{2} i-a^{2} \operatorname{sen}^{2} i}}{a \operatorname{sen}^{2} i}
$$

la quale dimostra che la linea sferica $\Lambda$ è un piccolo cerchio.

La curva richiesta ̀̀ dunque una trajettoria sotto l'angolo costante $i$ delle generatrici di un cono circolare retto, il cui semiangolo al vertice è

$$
\operatorname{arctg}\left(\frac{\sqrt{\cos ^{2} i-a^{2} \operatorname{sen}^{2} i}}{a \operatorname{sen}^{2} i}\right) \text {. }
$$

2.) Si voglia determinare la curva definita dalle equazioni

$$
R=a \cdot \operatorname{sen}\left(\frac{s}{a}+b\right) ; \quad \rho=\frac{a \operatorname{tg}\left(\frac{s}{m}+n\right)}{\sqrt{4 \operatorname{tg}^{2}\left(\frac{s}{n}+n\right)+\operatorname{sen}^{2}\left(\frac{s}{a}+b\right)}},
$$

con $a, b, m, n$ costanti.

Applicando la (11) si ricava

$$
\tau=\operatorname{tg}\left(\frac{s}{m}+n\right)
$$


ma dalla (10) si ha con un' integrazione

$$
s=a \sigma+h,
$$

con $h$ costante arbitraria. Perciò $\tau$ viene dato in funzione di $\sigma$ dall' equazione

$$
\tau=\operatorname{tg}\left(\frac{a s}{m}+k\right)
$$

con $k$ costante arbitraria.

Calcolando con questa formola il raggio di torsione $r_{0}$ della curva sferica $\Lambda$, si ottiene

$$
r_{0}=\frac{1+\tau^{2}}{\frac{d \tau}{d \sigma}}=\frac{m}{a}
$$

quantità costante. $\mathrm{E}$ siccome dall' equazione che dà $R$ si ricava, in forza della (8)

$$
\rho_{g}=\frac{a}{2},
$$

si vede che per ottenere la curva in discorso, basta prendere sopra una sfera di raggio 1 una curva $\Lambda$ a torsione costante $\frac{a}{m}$ e avvolgere il piano di una circonferenza di raggio $\frac{a}{2}$ sopra la superficie conica che projetta $\Lambda$ dal centro della sfera, colla condizione che il centro della circonferenza si trovi nel vertice del cono.

\section{$\S 4$.}

La formola ricordata al numero precedento

$$
\rho=\frac{R \sqrt{1-R^{\prime 2}}}{1-R^{\prime 2}-R R^{\prime \prime}}
$$

serve a determinare la curva piana il cui raggio vettore, rispetto a un dato centro, è una funzione nota dell' arco.

In tale ipotesi l'integrazione della (12) conduce alla relazione più generale possibile fra l'arco della linea e il suo raggio vettore relativo a un polo qualsivoglia del suo piano.

Se per es. si tratta di una circonferenza di raggio $k$, la relazione della 
quale ora si parla è la seguente

$$
R=\sqrt{2 k(k-a)+2 k \sqrt{k(k-2 a)} \cdot \operatorname{sen}\left(\frac{s+m}{k}\right)},
$$

con $a$ ed $m$ costanti arbitrarie.

S'incontra una formola del tutto analoga alla (12) quando si cerehi di stabilire l'espressione della curvatura circolare di una linea piana, chiamando con tal nome il rapporto all' arco elementare dell'angolo infinitesimo di due cerchi passanti per un punto fisso e tangenti alla linea data in due punti infinitamente vicini.

Sia $O$ il punto fisso, che assumeremo come origine dei raggi vettori $R$; siano $O A, O B$ due raggi vettori consecutivi di $L, M$ ed $N$ i loro punti medi. Condotte le perpendicolari a questi raggi vettori nei punti $M, N$, queste incontrano le normali alla $L$ in $A$ e $B$ nei centri $A_{1}, B_{1}$ dei cerchi. Chiamando $R_{1}$ il raggio di questi cerchi, $L_{1}$ il luogo dei punti $A_{1}, B_{1}, \ldots$ e $d \theta$ l'angolo infinitesimo sotto il quale si segano i cerchi, angolo rappresentato da $A_{1} O B_{1}$, si hanno le equazioni

$$
\begin{gathered}
\cos \left(O \widehat{A} A_{1}\right)=\sqrt{1-\overline{R^{2}}, \quad R_{1}=O A_{1}=\frac{R}{2 \sqrt{1-R^{\prime 2}}},} \\
d s_{1}=d R_{1}^{2}+R^{2} \cdot d \theta^{2},
\end{gathered}
$$

dall'ultima delle quali si ricava

$$
\frac{d \theta}{d s}=\frac{1}{R_{1}} \sqrt{\left(\frac{d s_{1}}{d s}\right)^{2}-\left(\frac{d R_{1}}{d s}\right)^{2}} .
$$

Ma fra le coordinate $x, y$ di $A$ e quelle $x_{1}, y_{1}$ di $A_{1}$ hanno luogo le relazioni

$$
x_{1}=x+R_{4} \cos \lambda, \quad y_{1}=y+R \cos \mu,
$$

essendo $\lambda, \mu$ gli angoli fatti cogli assi coordinati dalla normale principale di $L$.

Deducendosi da queste

$$
\frac{d s_{1}}{d s}=\left(1-\frac{R_{1}}{\rho}\right)^{2}+\left(\frac{d R_{1}}{d s}\right)^{2},
$$

si avrà

$$
\frac{d \theta}{d s}=\frac{1}{R_{1}}-\frac{1}{\rho} .
$$

Ricordando infine i valori precedenti di $\rho$ e $R_{1}$ e indicando con $K$ il raggio 
di curvatura circolare (rappresentato dal rapporto $\left.\frac{d s}{d \theta}\right)$ si ha

$$
K=\frac{R \sqrt{1-R^{\prime 2}}}{1-R^{\prime 2}-R R^{\prime \prime}} .
$$

L'analogia delle formole (12), (13) è manifesta.

Applicazioni. 1.9) L'equazione $R^{\prime \prime}=0$ è caratteristica della spirale logaritmica; per tale curva dunque, e solamente per essa, si ha la relazione $\rho=K$.

2. ) Arendosi dalle precedenti equazioni

$$
\frac{1}{i}+\frac{1}{K}=\frac{2 \sqrt{1-R^{\prime 2}}}{R}
$$

se sarà

con a costante, avremo

$$
\frac{1}{1}+\frac{1}{K}=\frac{2}{a}
$$

$$
R=a \cdot \operatorname{sen}\left(\frac{s}{a}\right)
$$

equazione di una circonferenza.

Dunque: Se la somma della curvatura ordinaria e della curvatura circolare di una linea piana è costante, la curvatura circolare è nulla e la linea ¿̀ una circonferenza.

3.') Avendosi

$$
\frac{1}{K^{2}}-\frac{1}{\rho^{2}}=\frac{4 R^{*}}{R}
$$

se si avrà

$$
\frac{1}{K^{2}}-\frac{1}{f^{2}}=\frac{4}{a^{2}}
$$

con a costante, si avrà pure

con $m$ ed $h$ costanti.

$$
R=\frac{1}{2 h}\left(h^{2} e^{\frac{s}{a}}-m e^{-\frac{s}{a}}\right)
$$

Tale è l'equazione delle linee in cui è costante la differenza dei quadrati della curvatura circolare e della curvatura ordinaria.

Per $m=0$ si ha l'equazione

$$
R=\frac{h}{2} e^{\frac{k}{a}}
$$

che rappresenta l'inversa di una sviluppante di circonferenza quando il centro di questa sia considerato come polo. 


\section{$\S 5$.}

Siano $\rho, r, R$ il raggio di curvatura, di torsione e della sfera osculatrice di una linea $L$ tracciata sopra una superficie; $\rho_{g}$ il raggio di curvatura geodetica; 0 , $\varepsilon$ le inclinazioni del pinno osculatore e della sfera osculatrice sul piano tangente.

E noto che

$$
\cos \theta=\frac{\theta}{\theta g} .
$$

Inoltre il coseno dell'angolo formato dalla normale principale di $L$ colla retta che congiunge il punto che si considera di $L$ col centro corrispondente della

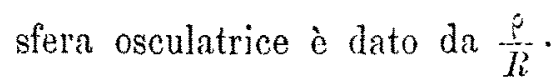

Applicando quindi anche la (14), si trova

$$
\cos \varepsilon=\frac{i}{R_{i} g}\left(\sqrt{\rho^{2} g-\rho^{2}}+r \frac{d \beta}{d s}\right)
$$

Facendo in quest' equazione successivamente $\varepsilon=\frac{\pi}{2}, \varepsilon=0$, si ricava:

Le condizioni necessarie e suffcienti perche le sfere osculatrici di una linea tracciata sopra una superficie siano ortogonali, ovvero tangenti, alla superficie sono rispettivamente che in ogni punto il raggio di curvatura geoletica $p_{g}$ della linea sia equale al raggio $R$ della sfera osculatrice, ovvero che sia legato a questo dalla relazione

$$
\frac{1}{p_{q}}=\frac{r}{R_{p}} \cdot \frac{d_{p}}{d s} \text {. }
$$

$\operatorname{Sin} L$ una linea a curratura non costante, tracciata sopra una superficie $S$. Quando $L$ sia linea di curvatura di $S$ e in ogni punto di essa si abbia $\varepsilon=\frac{\pi}{2}$, per un teorema altra volta da me dimostrato $\left(^{*}\right) L$ è una curva sferica. stante $p y$.

Risultando allora $R$ costante, per il teorema precedente risulta pure co-

Quando sia $\varepsilon=\frac{\pi}{2}$ e $\rho_{g}$ costante, per il teorema precedente sarà pure $R$

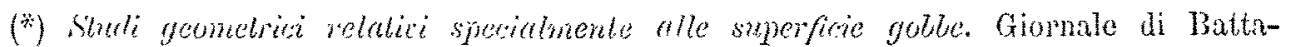
:lini, $188 \%$ 
costante; non essendo quindi la $L$ a curvatura costante (per dato), sarà sferica e conseguentemente linea di curvatura.

Quando infine $L$ sia linea di curvatura e $\rho_{g}$ costante, per un teorema del prof. Brioschi $\left(^{*}\right)$, la linea detta ¿̀ sferica e la sfera che la contiene è ortogonale alla superficie.

Dunque: Se per una linea tracciata sapra una superficie, e non a curvatura costante, sono verificate due delle proprietà seguenti:

$1 .^{a}$ che le sfere osculatrici siano ortogonali alla superficie,

2." che sia una linea di curvatura,

3. ${ }^{\mathrm{a}}$ che sia a curvatura geodetica costante,

la curva data è sferica e per essa è pure verificata la rimanente proprietà.

Quando $L$ sia una linea a curvatura costante e lungo di essa si abbia $\varepsilon=\frac{\pi}{2}$, la relazione precedente

$$
F_{g}=R
$$

dimostra che $\rho_{g}=\rho$, e che quindi $L$ è assintotica della superficie sulla quale è descritta. Se poi la $L$ è assintotica e lungo di essa si ha $\varepsilon=\frac{\pi}{2}$, la relazione $\rho_{g}=R$, che ora deve essere soddisfatta, dimostra che $\theta=R$, la quale relazione non può sussistere che per le linee a curvatura costante.

Se finalmente $L$ è assintotica e $\rho$ è costante, si ha $\frac{d \dot{t}}{d s}=0, \rho_{g}=\rho$ e quindi è soddisfatta la condizione $\rho_{g}=R$, d'onde segue che $\varepsilon=\frac{\pi}{2}$.

Perciò: Se una linea tracciata sopra una superficie possiede lue delle seguenti proprietà:

1. ${ }^{a}$ che le sfere osculatrici seghino la superficie ortogonalmente,

$2 .^{\mathrm{a}}$ che sia a curvatur' costante,

3. ${ }^{2}$ che sia assintotica, possiede pure la rimanente.

Quando $L$ sia a curvatura costante e lungo di essa si abbia $\hat{\varepsilon}=0$, dalla (16) si ricava $\frac{1}{\%}=0$, il che prova che $L$ è geodetica. stante.

Se poi $L$ è geodetica, e lungo di essa si ha $\Sigma=0$, la (16) dà $\rho=c 0-$

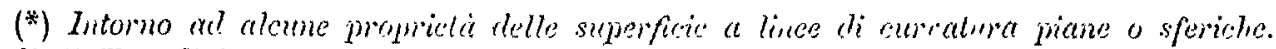
Amali di Tortolini, $185 \%$. 
Se infine è costante ed $L$ è geodetica, la (16) è soddisfatta identicamente, il che prova che $\varepsilon=0$.

Dunque: Se una linea tracciata sopra una superficie possiede due delle seguenti proprietì:

$1 .^{\mathrm{a}}$ che le sfere osculatrici siano tangenti alla superficie,

$2 .^{a}$ che sia a curvatura costante,

$3 .^{\text {a }}$ che sia geodetica, possiede pure la rimanente.

Quando la linea $L$ non sia geodetica delia superficie sulla quale è tracciata, dalle (14), (15) si può eliminare $\rho_{g}$ e risulta

$$
\cos \varepsilon=\frac{1}{R}\left(\rho \operatorname{sen} \theta+r \frac{d \hat{s}}{d s} \cos \theta\right) \text {. }
$$

Se in quest'equazione si suppone $p$ costante (nel qual caso $R=p$ ) si ottieno

$$
\cos \hat{\varepsilon}=\operatorname{sen} \theta, \text { d'ondo } \varepsilon+0=\frac{\pi}{2} \text {. }
$$

Dunque: Nelle linee a curvatura costante non piane e non geodetiche tracciate sopra una superficie qualunque, i piani osculatori e le sfere osculatrici segano la superficie sotto angoli complementari.

Risolvendo poi l'equazione (17) rapporto a coso, si ottiene

$$
\cos \theta=\frac{1}{l i}\left( \pm \rho \operatorname{sen} s+r \frac{d \theta}{d s} \cos s\right) \text {. }
$$

Per vedere quale dei due segni dobbiamo prendere in quest'equazione, si consideri la linea nella quale $\rho$ è costante. L'equazione precedente si riduce all' altra

$$
\cos \theta= \pm \operatorname{sen} \varepsilon
$$

e onde questa non sia in contraddizione con quanto è espresso dal teorema precedente, si deve scegliere il segno superiore. Abbiamo dunque in ogni caso

$$
\cos \theta=\frac{1}{l^{2}}\left(\rho \operatorname{sen} \varepsilon+r \cdot \frac{d p}{d s} \cos \varepsilon\right)
$$

Questa relazione è notevole, in quanto che si ottiene dalla (17) colla permutazione degli angoli $\theta$ ed $\varepsilon$.

Dividendo ambi i membri della (18) per $\rho$, si ha

$$
\frac{1}{i n}=\frac{\cos f}{i}=-\cdots \operatorname{sen} z+r \frac{d}{d s} \cdot \cos \varepsilon
$$


e quindi: Considerando una linea tracciata sopra una superficie qualunque, e deformando questa per flessione in un modo arbitrario, le quantità

rimangono invariate.

$$
\frac{\cos \theta}{\hat{i}}, \frac{\rho \operatorname{sen} s+r \frac{d \hat{\theta}}{d s} \cos s}{R_{i}},
$$

Si ricava ancora: Una geodetica e un'assintotica di una superficie qualunque sono caratterizzate rispettivumente dalle equazioni

$$
\begin{aligned}
& \rho \operatorname{sen} \varepsilon+r \frac{d s}{d s} \cos \varepsilon=0 \\
& \rho \cos z-r \frac{d s}{d s} \operatorname{sen} \varepsilon=0 .
\end{aligned}
$$

Si vede quindi che, nella deformazione per flessione di una superficie qualunque, l'equazione (19), relativa a una linea tracciata sopra di essa, è sempre soddisfatta ovvero non lo è mai; l'equazione (20) è invece soddisfatta tutto al più una sola volta per una speciale forma della superficie deformantesi.

Per una stessa linea $L$ si facciano passare due superficie $S, S_{1}$; siano $(\theta, \varepsilon),\left(G_{1}, \varepsilon_{1}\right)$ gli angoli dianzi considerati e relativi allo superficie $S$ ed $S_{1}$ rispettivamente. Se allora insieme alle equazioni (17), (18) si considerano le altre analoghe relative alle quantita $O_{1}, \varepsilon_{1}$, si può diro: Se, facendo passare due superficie $S, S_{1}$ per una stessa linea $L$, si trova verificata una delle relazioni

$$
\theta_{1}=\hat{z}, \quad \hat{\varepsilon}_{1}=\theta,
$$

si troverà pure verificata l'altra.

Siccome dall' equazione (17) si ricara

$$
\frac{\dot{p}^{\prime}}{i}=\frac{\operatorname{sen} \theta \cos \theta \pm \operatorname{sen} \varepsilon \cos s}{\cos ^{2} s-\cos ^{2} \theta} \cdot \frac{1}{i}
$$

le curve per le quali $\theta$ ed a sono due determinato funzioni doll'arco s di $L$, sono caratterizzate dalla relazione

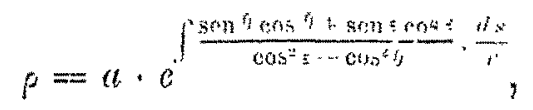

che lega fra loro l'arco o i raggi $p, r$.

Se poi

$$
\frac{\operatorname{sen} \theta \cos \theta \pm \operatorname{sen} z \cos z}{\cos ^{2} z-\cos ^{2} \theta}=k,
$$


con $k$ costante, la curva $L$ è caratterizzata dalla relazione

$$
\rho=a \cdot e^{k \int \frac{d s}{r}},
$$

Ia quale s'incontra anche quando si cercano le linee dello spazio nelle quali il raggio della sfera osculatrice è proporzionale al raggio del cerchio osculatore.

Osservando che la relazione (22) è soddisfatta quando $\theta$ ed $\varepsilon$ sono costanti e tenendo conto anche della (21), si può dire:

Se una linea tracciata sopra una superficie possiede due delle seguenti proprietì:

1. alle $i$ piani osculatori seghino la superficic sotto angolo costante,

2. che le sfere osculatrici seghino la superficie sotto angolo costante,

$3 .^{\mathrm{a}}$ che il raggio della sfera osculatrice sia proporzionale al raggio del cerchio osculatore, possiede pure la rimanente.

Si ha un nuovo teorema, se nell' enunciato precedente si sostituisce alla linea data un'elica, e se alla terza condizione si sostituisce l'altra che l' elica sia circolare o cilindrico-conica.

\section{$\S 6$.}

Delle curve (23), le quali s'incontrano in molte questioni, si può dare una facile costruzione.

Essendo $R>p$, si può porre

$$
\frac{p}{l i}=\cos i,
$$

ed $i$ sarà un angolo costante. Avremo allora

$$
r \rho^{\prime}=\rho \cdot \operatorname{tg} i
$$

se quindi $L_{0}$ è il luogo dei centri delle sfere osculatrici di $L$, sarà

$$
\begin{gathered}
\left.\rho_{0}=\rho+r\left(r^{\prime}\right)^{\prime}=\frac{\rho}{\cos ^{2} i} ; \quad r_{0}=\frac{\rho}{r} \mid \rho+r\left(r \rho^{\prime}\right)^{\prime}\right)=\frac{p^{2}}{r \cos ^{2} i} \\
\frac{l l s_{0}}{d s}=\frac{\rho}{r}+\left(r^{\prime} \rho^{\prime}\right)^{\prime}=\frac{\rho}{r \cos ^{2} i} .
\end{gathered}
$$

Da queste equazioni si deducono facilmente le altre

$$
\rho=\rho_{0} \cos ^{2} i ; \quad r=\frac{\rho^{2} 0}{r_{0}} \cos ^{2} i ; \quad R=\rho_{0} \sqrt{1+\rho_{0}{ }^{2}} \cdot \cos ^{2} i ;
$$


e poichè per la curva $L$ sussiste la relazione

$$
\rho=R \cos i
$$

per la linea $L_{0}$ sussiste l'altra

dalla quale si ricava

$$
\frac{d s_{0}}{d s_{0}}=\operatorname{tg} i
$$

con $a$ costante.

$$
\rho_{0}=s_{0} \cdot \operatorname{tg} i+a,
$$

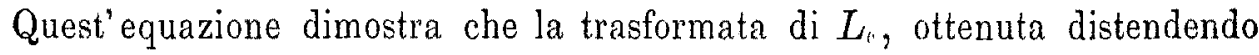
sopra di un piano la sua sviluppabile osculatrice, è una spirale logaritmica segante i raggi vettori uscenti dal polo sotto l'angolo $\frac{\pi}{2}-i$.

Se ora indichiamo con $L_{1}$ il luogo dei centri di curvatura di $L$, e con $A_{0}, A_{1}, A$ punti corrispondenti sulle tre curve $L_{0}, L_{1}, L$, avremo le equazioni

$$
A_{0} A_{1}=r \rho^{\prime}=\rho_{0} \operatorname{sen} i \cdot \cos i, \quad A_{1} A=\rho=\rho_{0} \cos ^{2} i,
$$

le quali servono per passare dalla linea $L_{0}$ (facilmente costruibile) alla $L_{i}$, e da questa alla $L$.

Nel terminare questa Nota, esporrò una costruzione assai facile delle sviluppabili che ammettono una geodetica sferica.

Se $L$ è una linea tracciata sopra una sfera qualunque $S$, la linea $L_{0}$ luogo degli estremi dei quadranti geodetici tangenti ad $L \grave{e}$, sulla sfera $S$, l'indicatrice delle tangenti di $L$. L'evoluta sferica $L_{1}$ di $L$ è una curra geodeticamente parallela ad $L_{0}$ e distante da essa di un quadrante geodetico. Ne segue che l'evoluta sferica $L_{2}$ di $L_{0}$ è la seconda evoluta sferica di $L$.

Perciò: In una linea sferica qualunque $L$ l'indicatrice dello spigolo di regresso della sviluppalile rettificatrice è la seconda evoluta sferica $L_{2}$ di $L$.

In base a questo teorema, per costruire una sviluppabile arente una geodetica tracciata sopra una sfera $S$, e il cui spigalo di regresso è, sopra $S$, una determinata linea $L_{2}$, si costruisca un'evolvente geodetica qualunque $L_{1}$ di $L_{2}$ e poscia un'evolvente geodetica qualunque $L$ di $L_{1}$. Indi per i punti di $L$ si conducano delle parallele ai raggi della sfera che vanno ai punti corrispondenti di $L_{2}$.

Queste rette sono le generatrici della sviluppabile domandata ed $L$ ne è la geodetica sferica.

Pama, semaio 189: 